\title{
Image of local density of states fluctuations in disordered metals in the differential conductance of tunneling via a resonant impurity level
}

\author{
Vladimir I. Fal'ko \\ School of Physics and Chemistry, Lancaster University, LA1 4YB Lancaster, United Kingdom
}

(Received 2 December 1996)

\begin{abstract}
Differential conductance of the resonant-tunneling structure with a single impurity level studied in the current plateau regime undergoes fluctuations around a zero average manifesting the energy dependence of the local density of states in a disordered electrode. Although the rms value of $d I / d V$ depends on disorder and barrier transparencies, it is almost independent of temperature and, as a function of bias voltage, has a correlation function scaled by the intrinsic width of the resonance, which can be regarded as a tool to measure this quantity beyond the main differential-conductance peak. The analysis is extended to the regime of classically high magnetic fields where both the amplitude and correlation magnetic field are expected to increase. [S0163-1829(97)01823-7]
\end{abstract}

In the recent vertical transport experiments on small-area double-barrier semiconductor structures, ${ }^{1-5}$ the resonant tunneling through a single impurity level created below the quantum well subband by a fluctuation in the density of charged donors has been observed and identified. Besides a possibility to study a number of interesting phenomena such as the Fermi-edge singularity in the two-dimensional electron gas $^{2}$ or the anticrossing of many-body levels of the impurity itself, ${ }^{3}$ a discrete impurity level can be also used for investigating the electron states in the electrodes, ${ }^{6,5}$ in particular, the mesoscopic fluctuations of the local density of states (LDOS) in them., ${ }^{7,8}$

Theoretically, the LDOS fluctuations in disordered metals were studied by Lerner $^{7}$ and shown to have a broad distribution $^{7,8}$ even in the metallic regime $\left(p_{F} l \gg 1\right)$. They are mainly governed by the fluctuations in the local structure of electronic wave functions in a disordered metal, ${ }^{9,10}$ rather than by the fluctuations of the number of states ${ }^{11,12}$ in a finite-size system. The manifestation of the density of states fluctuations in the transport experiments were discussed, first, in the context of the nonresonant tunneling between two disordered metals. ${ }^{13}$ Then, this idea has been extended ${ }^{14}$ to the studies of the resonant tunneling processes involving a resonance level in the barrier, and a contribution of LDOS fluctuations to the conductance of such a device was discussed, but only in the linear response regime. Under the experimental conditions of Refs. 1-5, the linear regime was hardly relevant, since, at a zero bias, the energy of a discrete impurity level, $E_{0}$ does not initially coincide with the bulk chemical potential $\mu_{l}$ coming to the resonance only after a bias voltage reaches the threshold value $V_{0}\left(E_{0}\right)$. Being essentially nonlinear, the current-voltage $(I(V))$ characteristics of such a device can be divided into three typical intervals: ${ }^{2-6}$ below the threshold, where $I \approx 0$; the threshold regime $V=V_{0}\left(E_{0}\right) \pm(\Gamma / a e)$, where $I(V)$ undergoes a jump after the resonant level crosses the Fermi level $\mu_{l}$ in the emitter; and the interval of a plateau, $V_{0}\left(E_{0}\right)<V<V_{1}\left(E_{1}\right)$, where the current remains nearly constant until the next impurity level $E_{1}$ is lowered enough to contribute to the transport.

The present paper is devoted to the quantitative analysis of features of the resonant-tunneling current in the plateau regime, where the interference effects in the bulk reservoirs dominate in the differential conductance $d I / d V$ giving rise to its irregular oscillations around a zero average. Under the described conditions, the differential conductance follows the form of the derivative of the LDOS fluctuation with respect to the energy. That enhances the contribution to the latter quantity coming from the finest energetic scale which can be resolved in such a system, that is the "spectrometer" width $\Gamma$. As a result, the correlation parameters of the differential conductance fluctuations are strictly bound to the energetic width of the resonant impurity level: The correlation voltage can be estimated as $\Gamma / e$, and the correlation magnetic field is that which provides a magnetic flux quantum per the area $L_{\Gamma}^{2}$, where $L_{\Gamma}=\sqrt{D h / \Gamma}$ is the diffusion length in a disordered metal corresponding to the lifetime $h / \Gamma$ of an electron in the resonant impurity state.

The amplitude of irregular oscillations of $d I / d V$ found in the calculations below is also related to the resonance width. The variance (i.e., the mean square) of the differential conductance at $V>V_{0}+\Gamma / e$ normalized by the height of the main resonance peak at $V=V_{0}$ is inverse proportional to conductance $g\left(L_{\Gamma}\right)$ of a piece of an electrode with dimensions $L_{\Gamma}^{d}$ measured in units of $e^{2} / h$ which can be interpreted on the basis of statistics of single-particle wave functions of a disordered metal. The value of the current in the plateau regime is determined by the sum of local densities (at the spectrometer position) of the wave functions $\left|\psi_{\epsilon}\left(r_{0}\right)\right|^{2}$ which have energies the $\epsilon$ within an energy interval $\Gamma$ around $E_{0}$ in a piece of a metal with characteristic dimensions $L_{\Gamma}$. These are the states which can contribute resonantly to the tunneling current. The average value of this sum is proportional to their total number $N(\Gamma)$ and to a typical density of a single state, $\left\langle\left|\psi_{\epsilon}\left(r_{0}\right)\right|^{2}\right\rangle \sim 1 / L_{\Gamma}^{d}$. Having been achieved over a step with the width $V_{\Gamma}=(\Gamma / a e)$, such a value of a current plateau results in the height of the main differential conductance peak $G_{\Gamma}$ which is proportional to $N(\Gamma)\left\langle\left|\psi_{\epsilon}\left(r_{0}\right)\right|^{2}\right\rangle / V_{\Gamma}$. On the other hand, $\psi_{\epsilon}\left(r_{0}\right)$ taken from an individual state is a random variable with dominantly Gaussian statistics in the metallic regime, ${ }^{10}$ so that $\operatorname{var}\left[\left|\psi_{\epsilon}\left(r_{0}\right)\right|^{2}\right] \sim\left\langle\left|\psi_{\epsilon}\left(r_{0}\right)\right|^{2}\right\rangle^{2}$. The variance of a sum of a large number, $N(\Gamma) \gg 1$, of random additives $\left|\psi_{\epsilon}\left(r_{0}\right)\right|^{2}$ is of the order of $N(\Gamma)\left\langle\left|\psi_{\epsilon}\left(r_{0}\right)\right|^{2}\right\rangle^{2}$, and being individual for each next energy interval $\Gamma$, this fluctuation is responsible for the fluctuation in the differential conductance with the variance 
proportional to $N(\Gamma)\left\langle\left|\psi_{\epsilon}\left(r_{0}\right)\right|^{2}\right\rangle^{2} / V_{\Gamma}^{2}$. Following Thouless, ${ }^{15}$ we estimate $N(\Gamma) \sim g\left(L_{\Gamma}\right)$, which gives that estimation mentioned on the top of this paragraph, $\left\langle\delta G^{2}\right\rangle / G_{\Gamma}^{2} \approx g^{-1}\left(L_{\Gamma}\right)$. It is worth mentioning that both the correlation parameters and the amplitude of the pattern of $d I / d V$ are insensitive to the temperature of electrons, since all the events we discuss here happen deep below the Fermi level, but they can be easily destroyed by the voltage noise with the amplitude larger than $\Gamma$.

To make the following quantitative analysis closer to the experimental conditions of Refs. 2-5, we assume that the emitter barrier is much stronger than the collector one. ${ }^{16}$ If so, the width of the resonance, $\Gamma$ is dominated by the electron escape to the collector, $\Gamma=\Gamma_{r}+\Gamma_{l} \approx \Gamma_{r}$, whereas the value of the current step is mainly determined by the tunneling rate $\Gamma_{l}$ through the thick barrier on the emitter side. Under this approximation, ${ }^{17}$ the current can be represented in the form ${ }^{18,14}$

$$
I(V) \approx \frac{e}{h} \int_{-\infty}^{\infty} \frac{\Gamma_{r}(\epsilon) \Gamma_{l}(\epsilon)\left[f_{l}(\epsilon)-f_{r}(\epsilon)\right] d \epsilon}{\left[\epsilon-E_{0}(V)\right]^{2}+\frac{1}{4} \Gamma^{2}(\epsilon)},
$$

where $f_{l(r)}(\epsilon)=\left\{1+\exp \left[\left(\epsilon-\mu_{l(r)}\right) / T\right]\right\}^{-1}$. Having been averaged over disorder, the $I(V)$ characteristics at the threshold can be described by the height of the resonance conductance peak at the voltage $V_{0}$ providing $\mu_{l}=E_{0}(V)$,

$$
\langle d I / d V\rangle_{\max } \approx G_{\Gamma}=\left(4 a e^{2} / h\right)\left(\Gamma_{l} / \Gamma\right),
$$

and its width at the half maximum, $V_{\Gamma} \approx \Gamma / e a$. The factor $a<1$ in Eq. (1) stands for taking into account an actual distribution of the potential drop across the structure. In the plateau regime (at $T<\mu_{l}-E_{0}$ ) the average current, $\langle I\rangle$ saturates at

$$
\left\langle I\left(\mu_{l}-E_{0}>\Gamma\right)\right\rangle \rightarrow 2 \pi e \Gamma_{l} / h=(\pi / 2) G_{\Gamma} V_{\Gamma},
$$

so that the disorder average of $\langle d I / d V\rangle$ tends to take a zero value.

The latter statement is made to stress that in the regime of bias voltages providing $E_{1}>\mu_{l}>E_{0}>\mu_{r}$, the differential conductance of the device is dominated by an irregular sample-specific energy dependence of the tunneling coupling between an impurity and the continuum of the electron states, e.g., in a disordered emitter "deep below" the Fermi level $\mu_{l}$,

$$
\Gamma_{l}(\boldsymbol{\epsilon})=2 \int \frac{d \mathbf{p} d \mathbf{p}^{\prime}}{(2 \pi)^{2 d}} \operatorname{Im}\left[G_{\epsilon}^{A}\left(\mathbf{p}, \mathbf{p}^{\prime}\right)\right]=t(\mathbf{p}) t^{*}\left(\mathbf{p}^{\prime}\right),
$$

where $t(\mathbf{p})$ is the tunneling matrix element between the impurity and bulk electrode, ${ }^{19}$ and $\operatorname{Im}\left[G^{A}\right]=\frac{1}{2}\left[G^{A}-G^{R}\right]$ are the exact retarded (advanced) Green functions of the electron in a bulk taken for a fixed configuration of disorder. Here, we focus only on the emitter dominated fluctuations, not only for quantitative reasons resulting from the assumed asymmetry of a structure, $\Gamma_{l} \ll \Gamma_{r}$, but also because at high enough voltages $^{2,4,5}$ the electron-electron collisions and emission of plasmons and optical phonons in the collector are fast enough to wash out quantum interference effects.

To be described quantitatively, a random energy dependence $\Gamma_{l}(\epsilon)$ and, therefore, the sample-specific fine structure of a current on the top of the plateau should be characterized by the disorder-averaged correlation function, of currents measured at different voltages, $\left\langle\delta I(V) \delta I\left(V^{\prime}\right)\right\rangle$, or at slightly different magnetic fields, $\langle\delta I(B) \delta I(B+\Delta B)\rangle$. In an infinite metallic systems $\left(p_{F} l \gg 1\right)$ with a continuous energy spectrum, the application of the standard diagrammatic perturbation theory methods is eligible (in contrast to closed systems where more vigorous techniques are required. ${ }^{11}$ ) Therefore, following, ${ }^{8,7,14}$ the current-current correlation function, e.g., $\left\langle\delta I(V) \delta I\left(V^{\prime}\right)\right\rangle$ can be represented in the form

$$
\begin{aligned}
\left\langle\delta I(V) \delta I\left(V^{\prime}\right)\right\rangle= & \frac{e^{2}}{(2 \pi)^{3} \nu \tau^{2}} \int d \omega d \epsilon \prod_{ \pm} \\
& \times \frac{\Gamma}{\left(\epsilon \pm \frac{\hbar}{2} \omega-E_{0} \mp \frac{1}{2} \Delta\right)^{2}+\frac{1}{4} \Gamma^{2}} \\
& \times \int \frac{d \mathbf{q}}{(2 \pi)^{d}}\left[P_{\omega}^{d(c)}(\mathbf{q})+P_{-\omega}^{d(c)}(\mathbf{q})\right] \\
& \otimes \prod_{n=1,2} \int \frac{d \mathbf{p}_{n}}{(2 \pi)^{d}} t\left(\mathbf{p}_{n}+\frac{\mathbf{q}}{2}\right) t^{*}\left(\mathbf{p}_{n}-\frac{\mathbf{q}}{2}\right) \\
& \times\left\langle G_{\epsilon}^{A}\left(\mathbf{p}_{n}+\frac{\mathbf{q}}{2}\right)\right\rangle\left\langle G_{\epsilon}^{R}\left(\mathbf{p}_{n}-\frac{\mathbf{q}}{2}\right)\right\rangle,
\end{aligned}
$$

where $\quad \omega=\left(\epsilon^{\prime}-\epsilon\right) / \hbar, \quad \Delta=E_{0}^{\prime}-E_{0} \approx a e\left(V^{\prime}-V\right)$, $\tau$ is the electron transport time in the emitter, $\left\langle G_{\epsilon}^{R(A)}(\mathbf{p})\right\rangle=[\epsilon-\epsilon(p) \pm(i \hbar / 2 \tau)]^{-1}$ is the average retarded $(R)$, and advanced $(A)$ single-electron Green functions, and $P^{d(c)}$ is the diffusion propagator in the diffuson and cooperon channels. In the above equation and wherever it is possible in the following analysis, we also use the inequality $\Gamma_{l} \ll \Gamma_{r}$ for a substitution $\Gamma_{r} \approx \Gamma$.

The analytic structure of the correlator in Eq. (4) and its meaning are very close to that of correlators of the integral density of states studied in Ref. 12 and of the level-level correlation functions studied in detail in quantum chaology. The expression in Eq. (4) contains, in principle, complete information about the electron dynamics in a disordered sample at any range of distances. Information about the electron behavior at the long-length scale is implicit into the diffusion poles of the correlation function $P^{d(c)}$ in the domain of $q<l^{-1}$. The integral multiplier behind it provides an ultraviolet cutoff of the integration, so that the result is sensitive to the electron dynamics at short distances near the resonant impurity. This creates an uncertainty in the estimation of the rms value of the current fluctuations observed using different techniques, and impedes a quantitative description of the observed features of the $I(V)$ characteristics from Refs. $2-5$, since a rigorous calculation of the currentcurrent correlator becomes model-dependent. (This is in contrast to the properties of spectral correlators studied in relation with the isolated disordered quantum dots ${ }^{11,20,21}$ which are universal in the zero-dimensional case.)

On the contrary, the finest structure of the energy dependence of the LDOS fluctuations and $I(V)$ characteristics formed due to the diffusive electron motion at the longlength scale can be enhanced by differentiation when the nonlinear conductance $d I / d V$ in the plateau region and its correlation functions, 


$$
\left\langle\left.\left.\frac{d I}{d V}\right|_{V} \frac{d I}{d V}\right|_{V^{\prime}}\right\rangle=\left\langle(\delta G)^{2}\right\rangle K_{d}\left(V^{\prime}-V\right)
$$

are studied. The correlation function in Eq. (5) can be obtained from Eq. (4) by taking the second derivative with respect to $\Delta=a e\left(V-V^{\prime}\right)$ and calculated only in the domain of $q<l^{-1}$ and $\epsilon-\epsilon^{\prime} \ll \hbar \tau^{-1}$, where the diffusion propagator $P_{\omega}^{d(c)}\left(\mathbf{r}, \mathbf{r}^{\prime}\right)$ is singular,

$$
P^{d(c)}=\int \frac{d \mathbf{q}_{\|}}{(2 \pi)^{2}} \int_{0}^{\infty} \frac{d q_{z}}{\pi} \frac{2 \cos \left(q_{z} z\right) \cos \left(q_{z} z^{\prime}\right) e^{i\left(\mathbf{r}_{\|}-\mathbf{r}^{\prime} \|\right) \mathbf{q}_{\|}}}{-i \omega+D q^{2}+\gamma},
$$

so that the integration over $\mathbf{p}, \mathbf{p}^{\prime}$ splits into two factorizing multipliers, each equal to $\left\langle\Gamma_{l}\right\rangle \tau / \hbar$ and independent of $\epsilon$ and $q$. Note that the diffusion propagator $P_{\omega}^{d(c)}\left(\mathbf{r}, \mathbf{r}^{\prime}\right)$ we need describes diffusion in a half space restricted by the tunneling barrier, so that $\left.\partial_{z} P^{d(c)}\right|_{z=0}=0$. One also should take its values close to the barrier itself which has to be taken into account when deriving the next formula. After integrating out the irrelevant variable $\epsilon$, two Lorentzian factors in Eq. (4) convolve into a single $\omega$-dependent kernel, and the correlation function in Eq. (5) takes the form

$$
\begin{aligned}
\left\langle\left.\left.\frac{d I}{d V}\right|_{V} \frac{d I}{d V}\right|_{V^{\prime}}\right\rangle= & -\beta^{-1}\left(\frac{\alpha e^{2}\left\langle\Gamma_{l}\right\rangle}{\pi \hbar}\right)^{2}\left(\frac{\partial}{\partial \Delta}\right)^{2} \\
& \times \int \frac{\Gamma \nu^{-1} d \omega}{(\hbar \omega-\Delta)^{2}+\Gamma^{2}} \int \frac{d \mathbf{q}_{\|}}{(2 \pi)^{2}} \\
& \times \int_{0}^{\infty} \frac{d q_{z}}{\pi} \frac{2\left[D q^{2}+\gamma\right]}{\omega^{2}+\left(D q^{2}+\gamma\right)^{2}} .
\end{aligned}
$$

In the case when the electrodes are $3 d$ metals (they extend in all directions over distances longer than $L_{\Gamma}=\sqrt{\hbar D / \Gamma}$ ), which is the case in the vertical tunneling devices grown without any undoped spacer in front of the tunneling barrier, the result of the integration takes the form of Eq. (5) with the variance

$$
\left\langle(\delta G)^{2}\right\rangle_{3}=\frac{\beta^{-1}}{8} \frac{\sqrt{\Gamma / \hbar D}}{\nu \hbar D} \frac{G_{\Gamma}^{2}}{\left[1+\frac{\hbar \gamma}{\Gamma}\right]^{3 / 2}},
$$

and correlation properties described by the function

$$
K_{3}=\frac{\left[2-\sqrt{1+\left(\frac{\Delta V}{\widetilde{V}_{\Gamma}}\right)^{2}}\right] \sqrt{1+\left[1+\left(\frac{\Delta V}{\widetilde{V}_{\Gamma}}\right)^{2}\right]^{1 / 2}}}{\sqrt{2}\left[1+\left(\Delta V / \widetilde{V}_{\Gamma}\right)^{2}\right]^{3 / 2}} .
$$

In Eq. (6), $\beta=1$ for the limit of a zero magnetic field, $\beta=2$ when $B L_{\Gamma}^{2}>\Phi_{0}$, and $\widetilde{V}_{\Gamma}=V_{\Gamma}+\hbar \gamma /(a e)$ is slightly modified, as compared to the width of the main resonance $V_{\Gamma}$ by the decoherence rate $\gamma$ of a floating-up " hole", below the Fermi level created in the emitter after the tunneling event. The value of the variance of fluctuations in Eq. (6) is normalized by the value $G_{\Gamma}$ from Eq. (1) in order to show how is it parametrically suppressed, as compared to the height of the main peak in $d I / d V$, by the factor of $\delta G / G_{\Gamma} \sim g^{-1 / 2}\left(L_{\Gamma}\right)$, where $g\left(L_{\Gamma}\right)$ is the conductance of a piece of an electrode with size $L_{\Gamma}$ in all directions scaled by $e^{2} / h$ [see the second multiplier in Eq. (6)].

A similar estimation is applicable to the case of a planar emitter. The analytical result for that case can be written as

$$
\begin{gathered}
\left\langle(\delta G)^{2}\right\rangle_{2}=\frac{\theta \beta^{-1}}{8 \nu \hbar D} \frac{G_{\Gamma}^{2}}{\left[1+\frac{\hbar \gamma}{\Gamma}\right]^{2}} ; \\
K_{2}=\frac{1-\left(\frac{\Delta V}{\widetilde{V}_{\Gamma}}\right)^{2}}{\left[1+\left(\frac{\Delta V}{\widetilde{V}_{\Gamma}}\right)^{2}\right]^{2}} .
\end{gathered}
$$

In Eq. (7), the factor $\theta$ distinguishes between two configurations of the device: $\theta=2$ for a "horizontal" tunneling through a lithographically processed barrier in a $2 d$ electron system, and $\theta=1$ for a "vertical" tunneling from a $2 d$ layer accumulated in front of the double-barrier structure in a device grown with a wide spacer. ${ }^{2}$ At larger voltages $\Delta V>V_{\Gamma}$, the result of Eqs. (6),(7) predicts a weak anticorrelation in the voltage-dependent pattern $d I / d V$.

Besides random variations as a function of a bias voltage, the total current and differential conductance should demonstrate random variations under the variation of a magnetic field-as any of mesoscopic effects. ${ }^{22,23}$ The correlation properties of random differential conductance oscillations under the variation of a magnetic field oriented along the current direction can be calculated after the diffuson in Eq. (4) is replaced by the solution of a generalized diffusion equation, $^{22}$

$$
\left[-i \omega-D\left(\nabla_{\|}-\frac{e}{\hbar c} \mathbf{A}\right)^{2}-D \partial_{z}^{2}\right] P_{\omega}^{d}\left(\mathbf{r}, \mathbf{r}^{\prime}\right)=\delta\left(\mathbf{r}-\mathbf{r}^{\prime}\right),
$$

where $\operatorname{rot} \mathbf{A}=\Delta \mathbf{B}$, complemented with the boundary condition $\left.\partial_{z} P^{d}\right|_{z=0}=0$. This has the form of

$$
\begin{aligned}
P_{\omega}^{d(c)}\left(\mathbf{r}, \mathbf{r}^{\prime}\right)= & \sum_{n=0}^{\infty} \int_{-\infty}^{\infty} \frac{d q_{y}}{2 \pi} \int_{0}^{\infty} \frac{d q_{z}}{\pi} \\
& \times \frac{2 \cos \left(q_{z} z\right) \cos \left(q_{z} z^{\prime}\right) \varphi_{n}\left(x, q_{y}\right) \varphi_{n}\left(x^{\prime}, q_{y}\right) e^{i q_{y}\left(y-y^{\prime}\right)}}{-i \omega+D q_{z}^{2}+2 D \lambda^{-2}\left(n+\frac{1}{2}\right)},
\end{aligned}
$$

where $\lambda^{-2}=e \Delta B / c \hbar$, and $\varphi_{n}\left(x, q_{y}\right)$ are the Landau wave functions of an electron in a magnetic field. As a result, we arrive at the correlation function of differential conductances, $K_{d}(\Delta B)$ in the form

$$
\begin{gathered}
K_{2}=\sum_{n=0}^{\infty} \frac{2 X^{-2}}{\left[n+\frac{1}{2}+\frac{1}{X}\right]^{3}}=-X^{-2} \psi^{(2)}\left(\frac{1}{X}+\frac{1}{2}\right), \\
K_{3}=\sum_{n=0}^{\infty} \frac{\frac{3}{2} X^{-3 / 2}}{\left[n+\frac{1}{2}+\frac{1}{X}\right]^{5 / 2}}, \quad X=\frac{2 e D \Delta B}{c(\Gamma+\hbar \gamma)},
\end{gathered}
$$


where $\psi^{(2)}(z)$ is the second order derivative from the $\psi$ function. At small $X \ll 1$, the correlation function $K_{d}(\Delta B)$ in Eq. (8) can be approximated by $K_{3} \approx 1-\frac{5}{32} X^{2}$ and $K_{2} \approx 1-$ $\frac{1}{4} X^{2}$. The characteristic correlation field $B_{c}$ (half width of the correlation function at half maximum) is

$$
B_{c}^{(d)} \approx c[\Gamma+\hbar \gamma] / e D \times \begin{cases}1.8, & d=3 \\ 1.3, & d=2 .\end{cases}
$$

The results of Eqs. (6), (7), (9) can be extended onto the differential conductance fluctuations in a classically strong magnetic field, $\omega_{c} \tau>1$. To achieve such a generalization, it is enough ${ }^{25}$ to replace the isotropic diffusion coefficient $D$ in expressions for the diffuson $P^{d}$ by a diagonal tensor $\operatorname{diag}\left(D, D_{\perp}, D_{\perp}\right)$ taking into account that the diffusion across the magnetic field direction is suppressed by the cyclotron motion, $D_{\perp}=D /\left[1+\left(\omega_{c} \tau\right)^{2}\right]$. When a magnetic field is oriented perpendicular to the tunneling barrier, the effect of skipping orbits ${ }^{24}$ does not affect the boundary condition to the equations on the diffuson (in contrast to conductance fluctuations in metallic wires ${ }^{26}$ ), so that we find that the variance of $d I / d V$ and the correlation parameter $B_{c}$ increase with a magnetic field as

$$
\frac{\left\langle(\delta G)^{2}\right\rangle_{B}}{\left\langle(\delta G)^{2}\right\rangle_{B=0}} \approx \frac{B_{c}(B)}{B_{c}(0)} \approx\left[1+\left(\omega_{c} \tau\right)^{2}\right] .
$$

The correlation voltage and the form of the correlation function $K_{d}(\Delta V)$ remain unchanged, since they are determined solely by the spectrometer width independently of a diffusion coefficient.

To summarize, the differential conductance of a system with the resonant tunneling from a disordered metal via a single impurity level was analyzed in the regime of a current plateau. In the systems of all dimensions we studied here, it fluctuates around zero average with the mean square value which scales with the height of the main resonance peak, $G_{\Gamma}$ and is inverse proportional to the conductance $g\left(L_{\Gamma}\right)$ (measured in quantum units) of a piece of a disordered electrode with typical dimensions of $L_{\Gamma} \sim \sqrt{\hbar D / \Gamma}$ determined by the width of the resonance itself: $\left\langle(d I / d V)^{2}\right\rangle \sim G_{\Gamma}^{2} / g\left(L_{\Gamma}\right)$. The value of the correlation magnetic field of fluctuations is also related to the length $L_{\Gamma}, B_{c} \sim \phi_{0} / L_{\Gamma}^{2}$ and correlation properties of the pattern of $d I / d V$ with respect to the voltage variations are found in the analytical form both in two and three dimensions as a function of a voltage scaled by the width of the main resonance peak. Both the amplitude of fluctuations and the correlation parameter $B_{c}$ are expected to increase with a magnetic field. As well as these fluctuations are the image of LDOS fluctuations deep below the Fermi level, their pattern is temperature independent, and they can be regarded as a tool to study the width of the impurityrelated level far beyond the main resonance tunneling conductance peak.

The author thanks A. Geim, L. Eaves, P. Main, and I. Lerner for discussions, and T. Schmidt and R. Haug for cooperation. This work was supported by EPSRC.
${ }^{1}$ B. Su, V.J. Goldman, and J.E. Cunningham, Science 255, 313 (1992); Phys. Rev. B 46, 7644 (1992).

${ }^{2}$ M.W. Dellow et al., Phys. Rev. Lett. 68, 1754 (1992); A.K. Geim et al., ibid. 72, 2061 (1994); P.J. McDonnell et al., Physica B 211, 433 (1995).

${ }^{3}$ M. Tewordt et al., Phys. Rev. B 46, 3948 (1992); 45, 14407 (1992); T. Schmidt et al., ibid. 51, 5570 (1995).

${ }^{4}$ M.R. Deshpande et al., Phys. Rev. Lett. 76, 1328 (1996); J.W. Sleight et al., Phys. Rev. B 53, 15727 (1996); Semicond. Sci. Technol. 9, 1919 (1994).

${ }^{5}$ T. Schmidt et al., Europhys. Lett. 36, 61 (1996).

${ }^{6}$ U. Sivan et al., Europhys. Lett. 25, 605 (1994).

${ }^{7}$ I.V. Lerner, Phys. Lett. A 133, 253 (1988).

${ }^{8}$ B. Altshuler, V. Kravtsov, and I. Lerner, in Mesoscopic Phenomena in Solids, edited by B.L. Altshuler, P. Lee, and R. Webb (North-Holland, Amsterdam, 1991), p. 449.

${ }^{9}$ F. Wegner, Z. Phys. B 36, 209 (1980); V. Fal'ko and K. Efetov, Europhys. Lett. 32, 627 (1995); Phys. Rev. B 52, 17413 (1995).

${ }^{10}$ V. Fal'ko and K. Efetov, J. Math. Phys. 37, 4935 (1996) and refs. therein.

${ }^{11}$ K.B. Efetov, Adv. Phys. 32, 53 (1983).

${ }^{12}$ B.L. Altshuler and B.I. Shklovskii, Zh. Éksp. Teor. Fiz. 91, 220 (1986) [Sov. Phys. JETP 64, 127 (1986)].

${ }^{13}$ Yu. Nazarov, Zh. Éksp. Teor. Fiz. 98, 306 (1990) [Sov. Phys. JETP 71, 563 (1990)]; A. Zyuzin and B. Spivak, ibid., 98, 1011 (1990) [71, 563 (1990)].

${ }^{14}$ I.V. Lerner and M.E. Raikh, Phys. Rev. B 45, 14036 (1992).

${ }^{15}$ D. Thouless, Phys. Rev. Lett. 39, 1167 (1977).
${ }^{16}$ In an asymmetric system with $t_{l} \ll t_{r}$, we can neglect the Coulomb blockade effect: see L.Y. Chen and C.S. Ting, Phys. Rev. B 44, 5916 (1991).

${ }^{17}$ For a while, we forget about the electron spin, but the final result will be written in such a form that this flaw will be automatically corrected-if there is no spin-orbit scattering in the bulk. Otherwise, the parameter $\beta$ in the answers should be taken as $\beta=4$.

${ }^{18}$ A. Chaplik and M. Entin, Zh. Éksp. Teor. Fiz. 67, 208 (1974) [Sov. Phys. JETP 40, 106 (1974)]; M. Azbel, Solid State Commun. 45, 527 (1983); W. Xue and P.A. Lee, Phys. Rev. Lett. 38, 3913 (1988).

${ }^{19}$ The tunneling matrix element can be roughly approximated as $t(\mathbf{p}) \approx \alpha i p_{z} \varphi_{0}\left(\mathbf{p}_{\perp}\right)$, which projects the wave function of a tunneling electron in the electrode onto the wave function of an impurity-related state in the double-barrier region.

${ }^{20}$ B. Simons and B. Altshuler, Phys. Rev. Lett. 70, 4063 (1993); Phys. Rev. B 48, 5422 (1993).

${ }^{21}$ C.W.J. Beenakker, Phys. Rev. Lett. 70, 4126 (1993); C.W.J. Beenakker and B. Rejaei, Physica A 203, 61 (1993).

${ }^{22}$ B.L. Altshuler and D.E. Khmelnitskii, Pis'ma Zh. Éksp. Teor. Fiz. 42, 291 (1985) [JETP Lett. 42, 291 (1985)].

${ }^{23}$ P.A. Lee and A.D. Stone, Phys. Rev. Lett. 55, 1622 (1985).

${ }^{24}$ A.K. Geim et al., Phys. Rev. Lett. 67, 3014 (1991); 69, 1248 (1992).

${ }^{25}$ S. Xiong and A.D. Stone, Phys. Rev. Lett. 68, 3757 (1992).

${ }^{26}$ D. Maslov and D. Loss, Phys. Rev. Lett. 71, 4222 (1993); D.E. Khmelnitskii and M. Yosefin, Surf. Sci. 305, 507 (1994). 